\title{
Detector energy calibration in the STEREO neutrino experiment
}

\section{Sergeyeva Viktoriya*t}

Laboratoire d'Annecy de Physique des Particules (LAPP)

E-mail: sergeyeva@lapp.in2p3.fr

The STEREO experiment was developed to investigate the hypothesis of a light sterile neutrino arising from the observed discrepancy between measured reactor antineutrino fluxes and revised flux predictions, known as the Reactor Antineutrino Anomaly.

The detector is located at $10 \mathrm{~m}$ from the compact nuclear reactor core of the Institut LaueLangevin. The segmentation of the detector target in 6 cells allows measuring the neutrino energy spectrum at different baselines. Antineutrino interactions are detected by the inverse $\beta$-decay reaction in Gd-loaded liquid scintillator.

STEREO started taking data in November 2016. About 70 days of data have been recorded during reactor operation and $\sim 25$ days during reactor shut down. STEREO plans to record $\sim 150$ days more in 2018.

To perform a correct energy reconstruction of neutrino events, the detector energy response must be determined accurately. Different radioactive gamma-ray sources, from 0.5 to $\approx 4.4 \mathrm{MeV}$, are used to constrain the non-linearity of the scintillator response. Calibration data analysis provides the relation between the charge detected by the photomultipliers and the true deposited gamma energy obtained by simulations. The main goal is to reconstruct the energy scale within a $2 \%$ uncertainty.

EPS-HEP 2017, European Physical Society conference on High Energy Physics 5-12 July 2017

Venice, Italy

${ }^{*}$ Speaker.

${ }^{\dagger}$ On behalf of the STEREO collaboration. 


\section{The STEREO experiment}

The scenario with an additional fourth (or more) light sterile neutrino was suggested in 1990s, after the report of anomalous event excess in the $\bar{v}_{\mu} \rightarrow \bar{v}_{e}$ appearance [1, 2] known as the LSND anomaly. In addition, GALLEX and SAGE experiments $[3,4]$ reported $\sim 23 \%$ deficit in measured activity of the ${ }^{51} \mathrm{Cr}$ neutrino source inside ${ }^{71} \mathrm{Ga}$-filled tanks. The interpretation of this "Gallium anomaly" and its compatibility with the Bugey and Chooz reactor short baseline data performed in [5] was a possible indication in favour of $\bar{v}_{e}$ disappearance by oscillations with parameters $\sin ^{2} 2 \Theta \gtrsim 0.03$ and $\Delta m^{2} \gtrsim 0.1 \mathrm{eV}^{2}$. Finally, the re-evaluation of the reactor $\bar{v}_{e}$ flux together with reactor experimental data at short baselines $(<100 \mathrm{~m})$ points to a deficit of $\approx 6 \%$ between the measured and predicted reactor $\bar{v}_{e}$ flux $[6,7]$ which is called the Reactor Antineutrino Anomaly (RAA).

In order to explain the RAA, the STEREO experiment is one of numerous very short baseline reactor experiments proposed all over the world. The scope of these experiments and their main features can be found in [8].

The STEREO experiment, proposed in 2013, is searching for a neutrino oscillation pattern in the energy spectrum of $\bar{v}_{e}$ emitted by the Institut Laue-Langevin (ILL) nuclear reactor in Grenoble. The ILL reactor has a compact core $(\Phi 40 \mathrm{~cm} \& H 80 \mathrm{~cm})$ loaded with highly enriched uranium (HEU) fuel at $93 \%$ of ${ }^{235} U$. Particularly high levels of $\gamma$ and neutron backgrounds are observed due to neighbouring reactor experiments on the ILL site. Thus, several layers of shielding surround the detector on the outside (concrete, $\mathrm{Pb}, B_{4} C$ and $\mathrm{PE}$ sheets). The detector itself also incorporates strong passive shielding $\left(\mathrm{Pb}\right.$, borated $\mathrm{PE}, B_{4} C$, mumetal) and an active muon detector on the top.

The internal structure of the detector consists of 2 main parts: the so-called Gamma Catcher (GC) and the target (fig. 1). The GC outer crown has 4 cells that collect energy escaping from the target and detect background coming from outside of the target. The target is segmented into 6 cells $\left(0.37 \times 0.9 \times 0.9 \mathrm{~m}^{3}\right)$ filled with Gd-loaded liquid scintillator (LS). In each cell, the goal is to reconstruct the detected $\bar{v}_{e}$ event and its energy. STEREO uses the Inverse Beta Decay (IBD) reaction $\bar{v}_{e}+p \rightarrow e^{+}+n$ which produces two signals correlated in time and distance.

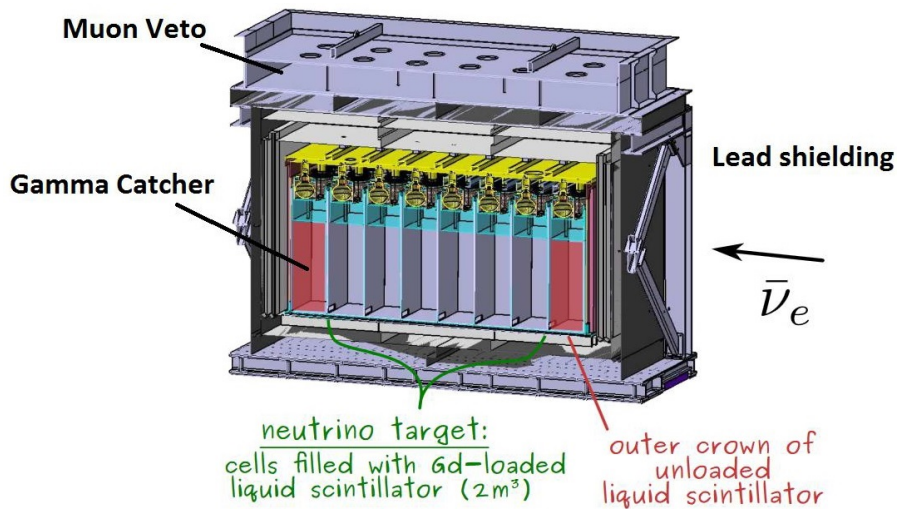

Figure 1: The STEREO detector.

The positron gives a prompt signal by almost instant energy deposit in the LS from the annihilation with an electron. After thermalisation, the neutron can be captured by $\mathrm{Gd}(n, \gamma)$ and 
generates a delayed signal corresponding to a $\gamma$ cascade with a total energy of $8 \mathrm{MeV}$ produced by the Gd de-excitation.

After 2 weeks of commissioning in November 2016, STEREO recorded data during 70 days of reactor ON and 28 days of reactor OFF till March 2017. Reactor shutdown started on March 2017 which is expected to last until 2018 due to maintenance and security issues. The STEREO detector was also in maintenance from March to October 2017. The detector will be able to take an important amount of reactor OFF data from October 2017 until beginning of 2018. STEREO expects to record $\sim 150$ days of reactor ON data in 2018 .

\section{Energy calibration}

STEREO aims to compare the measured antineutrino energy spectrum and number of events for the 6 target cells. Thus, each cell is calibrated independently despite possible event signal leaks between neighbouring cells. The energy response is expected to be known within a $2 \%$ uncertainty. The scintillation response, non uniformities and energy scale have to be measured and characterised precisely. The experiment is calibrated using radioactive gamma and neutron sources.

\subsection{Calibration method}

Calibration can be performed outside the Gamma Catcher and inside the target cells (fig. 2). During internal calibrations, radioactive sources are introduced via guide tubes into cells 1,4 and 6. During external calibrations, the sources can be moved along the detector perimeter, outside the GC crown, and they can also be moved underneath the detector cells. The automated system used for source transport along the STEREO perimeter is called pantograph and appears on figure 2 (left part) as the cross structure. In the vertical direction, sources are usually placed at three $\mathrm{Z}$ positions: top ( $\sim 80 \mathrm{~cm}$ from the cell floor), middle ( $\sim 45 \mathrm{~cm}$ from the cell floor) and bottom $(\sim 17 \mathrm{~cm}$ from the cell floor).
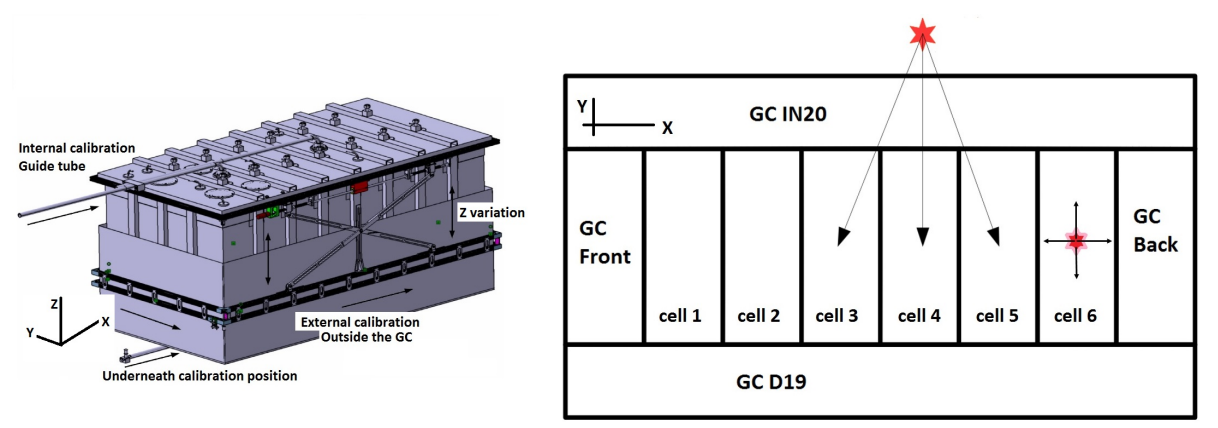

Figure 2: Calibration positions in STEREO (left) and cell identification in the horizontal plane (right).

Each target cell is readout on its top side by 4 photomultiplier tubes (PMTs). The GC is segmented in 4 cells, 2 short and 2 long ones. The short and long GC cells have 4 and 8 PMTs per cell respectively. The identification of cells is shown on figure 2. The GC front cell is facing the reactor.

The set of radioactive sources used for STEREO calibrations is presented in table 1. The energy computed by Monte Carlo simulation and used in calibration coefficient calculations is 
smaller by a few percent than its nominal value in table 1 due to Compton energy losses. For target cells this difference is smaller than $4 \%$. For Gamma Catcher long segments and external source this difference is $\sim 9 \%$ for central positions and $\sim 14 \%$ for corner positions. Smaller deposited energy in GC is due to the larger amount of inert material that photons can meet in comparison with target cells.

$$
\begin{array}{cccccccc}
\text { Source } & { }^{68} \mathrm{Ge} & { }^{137} \mathrm{Cs} & { }^{54} \mathrm{Mn} & { }^{65} \mathrm{Zn} & { }^{60} \mathrm{Co} & { }^{24} \mathrm{Na} & \mathrm{AmBe} \\
E_{\text {nominal }}(\mathrm{MeV}) & 2 \times 0.511 & 0.662 & 0.835 & 1.115 & 1.173 \& 1.332 & 1.369 \& 2.754 & 2.2,4.4
\end{array}
$$

Table 1: Radioactive sources used for calibrations in the STEREO experiment.

The main task is to translate the charge collected at the PMTs into energy. For that, we extract the charge, in photoelectrons, for a given source and position of calibration and we call it $Q_{\text {Data }}$ (pe). Monte Carlo simulation gives us the true deposited energy for this source and position, $E_{d e p}$ in $\mathrm{MeV}$. We define the calibration coefficient (CC) as the ratio of charge over deposited energy 2.1. The charge is selected by fitting a Gaussian to the observed peak's maximum and computing the average charge within a wide interval around the Gaussian mean value. In Monte Carlo, we extract the average deposited energy for the same charge selection applied to data.

$$
C C=\frac{Q_{\text {Data }}}{E_{\text {dep }}}
$$

Next, we present two detector features:

- the spatial detector response using calibration coefficients obtained with a ${ }^{54} \mathrm{Mn}$ source;

- the non-linearity into liquid response due to the quenching of scintillation light.

\subsection{Detector spatial response}

Vertical non-uniformity in detector response is present in the target and the GC. The amount of collected light depends on the distance between the interaction and the PMTs. The attenuation length of the liquid scintillator contributes as well to this difference.

Figure 3 shows the vertical non-uniformity of the collected light in the target with a ${ }^{54} \mathrm{Mn}$ source in cell 6 at 5 different heights. Values are normalized to the middle position. The charge varies from $-3 \%$ to $+3 \%$ between the bottom and the top positions.

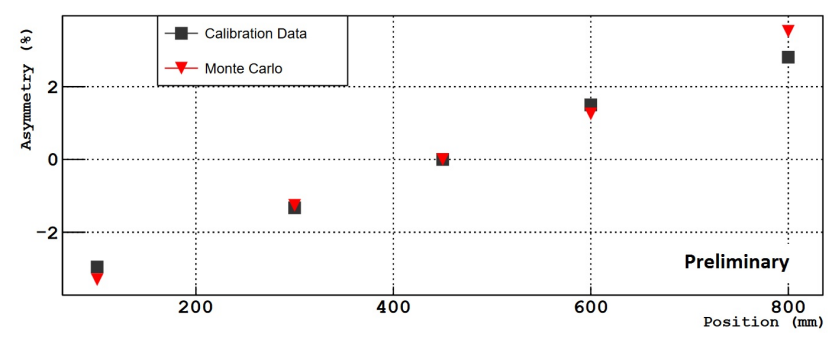

Figure 3: Vertical non-uniformity of the collected light in the target. The position is the distance from the source to the cell floor. 
Concerning the GC calibrated from the outside with a ${ }^{54} \mathrm{Mn}$ source, calibration coefficients vary from $\sim-2 \%$ to $\sim+2 \%$ between the bottom and the top for long cells and from $\sim-4 \%$ to $\sim+4 \%$ for the GC short cell. The collected charge verifies the order $Q_{t o p}>Q_{\text {middle }}>Q_{\text {bottom }}$.

Target cells response non-uniformity, in $\mathrm{X}$ and $\mathrm{Y}$ directions (axes are defined in figure 2), is small and not useful to correct. In contrast, for GC long cells the non-uniformity in X is significant. Figure 4 shows the distribution of ${ }^{54} \mathrm{Mn}$ calibration coefficients in function of the $\mathrm{X}$ position of an external source moving along the detector perimeter. The difference between central and edge calibration coefficients is $\sim 6-8 \%$. When the $\gamma$ source is in edge position, photons have more probability to escape outside.

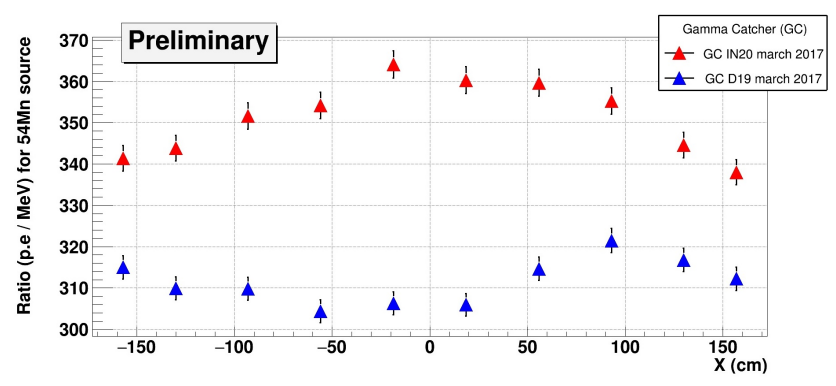

Figure 4: GC response non-uniformity in $\mathrm{X}$ for the long cells.

For short cells we do not have the resolution to reconstruct the vertex with precision whereas in the long sides of the GC it is possible to reconstruct the interaction vertex.

The work to adjust Monte Carlo to data results is being performed. At this stage, figure 5 shows a Monte Carlo and data comparison for the target charge produced by a ${ }^{54} \mathrm{Mn}$ source placed in cell 6 in middle $\mathrm{Z}$ position. Background is not removed on this plot.

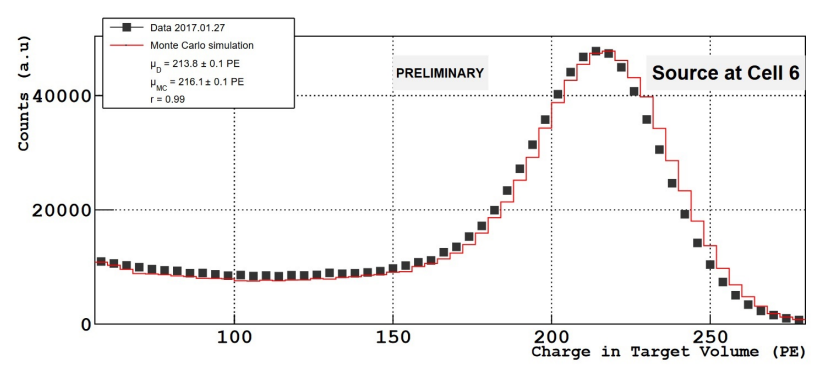

Figure 5: Data and Monte Carlo target charge distribution comparison for a ${ }^{54} \mathrm{Mn}$ source in cell 6.

Figure 6 shows the reconstructed energy for a ${ }^{54} \mathrm{Mn}$ source in cell 6 in middle $\mathrm{Z}$ position, for data and Monte Carlo. Background is removed here. In the energy reconstruction procedure, we are accounting for light produced in the cell and light leaking from other cells.

\subsection{Non linearity into scintillator liquid response}

The causes of the LS non-linearity are quenching, Cerenkov light emission and non-linearity given by the electronics. The dominant effect is the one coming from quenching and is the only factor discussed in this section. The effect of the electronics is expected to be under $1 \%$. 


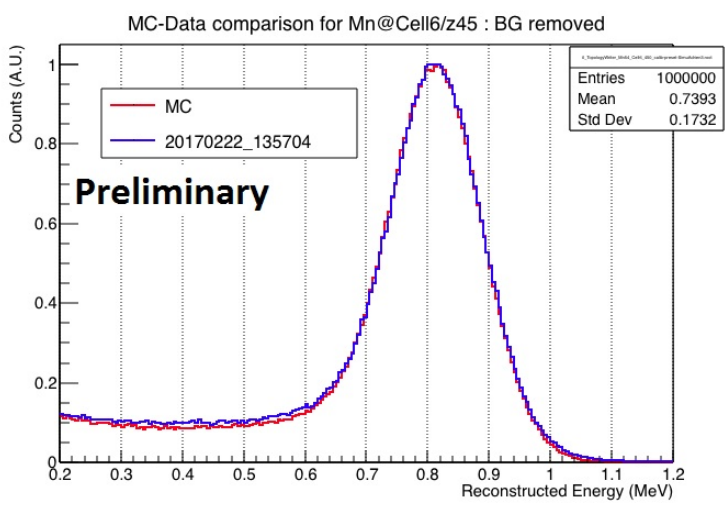

Figure 6: Reconstructed energy for a ${ }^{54} \mathrm{Mn}$ source in cell 6 in middle $\mathrm{Z}$ position.

The LS quenching depends on the particle and on its energy. Non-linearity is important for low energies and the signal presents a saturation behaviour for energies larger than $\sim 4.5 \mathrm{MeV}$.

Different mono- $\gamma$ and multi- $\gamma$ sources were placed inside cell 6 in middle $\mathrm{Z}$ position. Calibration coefficients were calculated for the neighbouring cell 5 (see fig. 2). When an AmBe source was used, it was placed in cell 4 because tubes in cells 4 and 5 are more separated than in cells 5 and 6. This additional distance helps us to reduce more the proton recoil effect produced by AmBe neutrons.

The LS response verifies $\frac{d L}{d x}=S \frac{d E}{d x}$ where $L$ is the LS light yield in $\frac{\gamma}{M e V}$ and $S$ is the LS efficiency. The non-linearity is described by the Birks coefficient, $K_{B i r k s}$ in $\frac{\mathrm{mm}}{\mathrm{MeV}}$ and verifies the Birk's law equation 2.2 [9].

$$
\frac{d L}{d x}=S \frac{\frac{d E}{d x}}{1+K_{\text {Birks } \frac{d E}{d x}}}
$$

Figure 7 shows the calibration coefficent variation with the energy of the source.

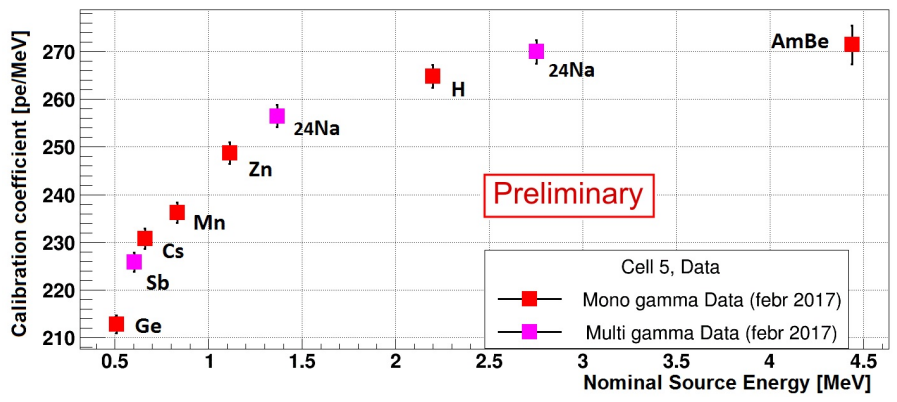

Figure 7: Quenching effect in the LS in cell 5 using different $\gamma$ sources in middle $\mathrm{Z}$ position.

For this preliminary quenching curve, errors are approximated to $\sim 1 \%$ for all sources except for AmBe, $\sim 1.5 \%$, due to neutron contamination. The next step of the calibration study will be to tune precisely Monte Carlo simulation parameters, such as the target light yield and the Birk's coefficient, in order to obtain the same quenching behaviour as in the data.

More information about the STEREO experiment can be found in [10, 11, 12, 13]. 


\section{Conclusions and prospects}

From November 2016 to March 2017, internal and external calibration runs, corresponding to 87 days of data taking, have been recorded. We have presented here the detailed calibration procedures of the STEREO experiment.

The detector response variation with the vertical coordinate $\mathrm{Z}$ using a ${ }^{54} \mathrm{Mn}$ source results in $3 \%$ differences between middle and top/bottom $\mathrm{Z}$ positions. The longitudinal response variation for the long Gamma Catcher cells gives a $6-8 \%$ difference in calibration coefficients between the central and edge positions. Monte Carlo adjustement to data is underway and gives first encouraging results for energy reconstruction using a ${ }^{54} \mathrm{Mn}$ source. Concerning the quenching effect in the target liquid scintillator, preliminary results show a $\sim 20 \%$ difference between calibration coefficients using ${ }^{68} \mathrm{Ge}$ and ${ }^{24} \mathrm{Na}$ sources.

A detailed study of systematics, a precise Monte Carlo tuning and the finalisation of energy reconstruction will be the next steps needed to perform accurate spectral analyses. Approximately 150 days of reactor ON data taking are expected during 2018.

\section{References}

[1] C. Athanassopoulos et al. Candidate Events in a Search for $\bar{v}_{\mu} \rightarrow \bar{v}_{e}$ Oscillations. Phys. Rev. Lett. Volume 75, Number 14, pages 2650 - 2653, 1995.

[2] A. Aguilar et al. Evidence for neutrino oscillations from the observation of $\bar{v}_{e}$ appearance in a $\bar{v}_{\mu}$ beam. Phys. Rev. D Volume 64, pages 112007 - 1 - 1120007 - 22, 2001.

[3] F. Kaether et al. Reanalysis of the Gallex solar neutrino flux and source experiments. Phys. Lett. B Volume 685, pages 47-54, 2010.

[4] J. N. Abdurashitov et al. Measurement of the solar neutrino capture rate with gallium metal. III: Results for the 2002-2007 data-taking period. Phys. Rev. C Volume 80, 015807, 2009.

[5] Mario A. Acero, Carlo Giunti, Marco Laveder. Limits on $v_{e}$ and $\bar{v}_{e}$ disappearance from Gallium and reactor experiments. Phys. Rev. D Volume 78, 073009, 2008.

[6] Th. A. Mueller et al. Improved predictions of reactor antineutrino spectra. Phys. Rev. C Volume 83, 054615, 2011.

[7] S. Gariazzo, C. Giunti, M. Laveder, Y. F. Li, E. M. Zavanin. Light sterile neutrinos. Journal of Physics G: Nuclear and Particle Physics, Volume 43, Number 3, 2016.

[8] P. Vogel, L. J. Wen, C. Zhang. Neutrino oscillation studies with reactors. Nature Communications 6, $6935,2015$.

[9] Glenn F. Knoll. Radiation detection and measurement. Second edition, ISBN 0-471-81504-7.

[10] S. Zsoldos et al. Search for a sterile neutrino with the STEREO detector at ILL. Proceedings of the 50th Rencontres de Moriond, Electroweak Interactions and Unified Theories 2015. arXiv:1602.00568

[11] V. Helaine et al. Sterile neutrino search at the ILL nuclear reactor: the STEREO experiment. Talk presented at NuPhys2015. arXiv:1604.08877

[12] L. Manzanillas et al. STEREO: Search for sterile neutrinos at the ILL. Proceedings of the NOW 2016 conference. arXiv:1702.02498

[13] V. Helaine et al. Electronics for the STEREO experiment. Proceedings of the Neutrino2016 conference. arXiv:1610.00003 